\title{
Pre-Sale Competition When Consumers Regret
}

\author{
Li Huang \\ School of Management, Jinan University, Guangzhou, China \\ Email: huangli3456@163.com
}

How to cite this paper: Huang, L. (2018) Pre-Sale Competition When Consumers Regret. American Journal of Industrial and Business Management, 8, 658-666. https://doi.org/10.4236/ajibm.2018.83044

Received: March 13, 2018

Accepted: March 23, 2018

Published: March 27, 2018

Copyright $\odot 2018$ by author and Scientific Research Publishing Inc. This work is licensed under the Creative Commons Attribution International License (CC BY 4.0).

http://creativecommons.org/licenses/by/4.0/

\begin{abstract}
This paper takes consumers anticipated regret into consideration in the pre-sale competition decision model, and analyzes the impact of anticipated regret on retailers' pre-sale price and revenue in the pre-sale context where consumers have uncertain valuations and preference matching. The results show that, for retailers with higher probability of matching, it is expected that anticipated buying regret is harm to improve revenue, while anticipated waiting regret is beneficial. Conversely, for the retailer with lower possibility of matching, anticipated buying regret is beneficial to improve revenue, while waiting regret is not.
\end{abstract}

\section{Keywords}

Anticipated Regret, Pre-Sale Competition, Retailers' Revenue

\section{Introduction}

Pre-sale is a form of advance selling, which refers to a marketing practice that a seller uses to induce buyers to commit to purchase a good before the time of consumption [1]. Many retailers presell new product before the time of release, such as Amazon, Target, Best Buy, and others [2]. As for consumers, they are guaranteed to obtain the new products at the time of release. However, in the pre-sale period, consumers lack sufficient information to determine the product valuations, and even to determine whether the product's property matches their own preferences [3]. Only until the second period, can they know the product valuation and preference matching. Therefore, when consumers purchase the product in the first period, if he obtains the product in second period and finds that the actual product valuation is lower, or the product does not match his preference, he will regret buying the product in advance. But when he chooses to wait in the first period, after the uncertainty is eliminated in the second period, he will regret waiting because of missing the opportunity to purchase the suitable 
product in advance. Consumers anticipate the regret as a result of decision-making, and determine when to buy a product and which product to buy. Therefore, this behavior will also affect the retailer's pre-sale strategy.

Consumer regret has been studied by many scholars. Many experimental studies have shown that, because of uncertainty, the anticipated regret of the decisionmaking results will have an impact on individual behavioral decisions, in particular the purchase timing [4] [5]. In addition, Diecidue et al. studied the influence of consumer regrets on purchase decisions in a dynamic buying environment [6], pointing out that the consumers who are disgusted with inaction regret will tend to be more inclined to purchase in advance, otherwise, they will delay purchase. Nasiry and Popescu successfully described the effects of the "action regret" and "inaction regret" on the consumers buying decisions and the monopoly's pre-sale strategy [7], which provided reference for companies to formulate sales strategies. Chao, Liu and Zhan analyzed the influence of consumers with different degrees of regret on the competitive firms pricing and mixed probability of vertical probability selling [8]. Based on that paper, Jiang et al. further proposed consumer utility functions with consideration of anticipated regret, and analyzed the influence of anticipated regret sensitivity and consumer heterogeneity on the R \& D strategy of new products [9].

In particular, Nasiry and Popescu have studied the impact of consumers' anticipated regret behaviors in the context of advance selling, which focuses on the monopoly firm. Based on their study, our article models the consumer's utility function with anticipated regret in the pre-sale competition context, and analyses the influence of consumer anticipated regret on the retailers' pre-sales strategies and revenues. With analytical framework, this paper might help pre-sale retailers to understand the influence of consumer anticipated regret on revenues, and to respond better to regret in pre-sale competition context where consumers face valuation and matching uncertainty.

\section{The Model Setup}

There are two retailers, A and B, competing in the market. Both companies sell similar products at the pre-sale stage and spot selling stage with unit cost that we normalize to zero. The pre-sale price is $p_{1 i}$, and the spot sellling price is $p_{2 i}$. The retailers simultaneously announce $p_{1 i}$ at the pre-sale stage and $p_{2 i}$ at the spot selling stage. Without capacity constraints, each retailer maximizes the revenue of the whole selling period by establishing optimal pre-sale price and spot selling price.

Forward-looking consumers enter the market at the beginning of the pre-sale stage with certain market size normalized to one. At the pre-sale stage, consumers have uncertainty product valuations and preference matching, and those uncertainty will be eliminated at spot selling stage. The utility obtained by purchasing one product from retailers is $u_{t i}=v f_{i}-p_{t i}, f_{i}=\{0,1\}, f_{i}=1$ indicates match, and $f_{i}=0$ indicates no match. Consumers are uncertain 
about the product valuation and preference matching in the first period, but know the distributions with $\operatorname{Prob}\left(v=v_{h}\right)=\operatorname{Prob}\left(v=v_{l}\right)=\frac{1}{2}$ and $\operatorname{Prob}\left(f_{A}=1, f_{B}=0\right)=\alpha, \operatorname{Prob}\left(f_{A}=0, f_{B}=1\right)=1-\alpha$. The closer $\alpha$ is to 0 or 1 , the lower the uncertainty of preference matching is, and the closer $\alpha$ is to $\frac{1}{2}$, the higher the uncertainty of preference matching is. Being forward-looking, customers can correctly predict that the spot period prices are equal to $v_{h}$. To simplify the calculation, we suppose $\frac{v_{l}}{v_{h}} \leq \frac{1}{4}$.

\subsection{Consumer Utility Functions with Anticipated Regret}

According to Jiang's consumer utility function with anticipated regret, the expected utility available to consumers for purchasing from $\mathrm{A}$ in the first period is:

$$
\begin{aligned}
E\left(u_{1 A}\right)= & \alpha E(v)-p_{1 A}-\rho\left\{E\left(\left[\max \left(0-p_{1 B}, v-p_{2 A}, 0\right)-\left(v-p_{1 A}\right)\right]^{+}\right)\right. \\
& \left.+E\left(\left[\max \left(v-p_{1 B}, v-p_{2 B}, 0\right)-\left(0-p_{1 A}\right)\right]^{+}\right)\right\}
\end{aligned}
$$

The expected utility for purchasing from B in the first period is:

$$
\begin{aligned}
E\left(u_{1 B}\right)= & (1-\alpha) E(v)-p_{1 B}-\rho\left\{E\left(\left[\max \left(0-p_{1 A}, v-p_{2 B}, 0\right)-\left(v-p_{1 B}\right)\right]^{+}\right)\right. \\
& \left.+E\left(\left[\max \left(v-p_{1 A}, v-p_{2 A}, 0\right)-\left(0-p_{1 B}\right)\right]^{+}\right)\right\}
\end{aligned}
$$

And the expected utility for waiting in the first period is:

$$
\begin{aligned}
E\left(u_{W}\right)= & \alpha E\left[v-p_{2 A}\right]^{+}+(1-\alpha) E\left[v-p_{2 B}\right]^{+} \\
& -\sigma\left\{E\left(\left[\max \left(v-p_{1 A}, 0-p_{1 B}\right)-\max \left(0, v-p_{2 A}\right)\right]^{+}\right)\right. \\
& \left.+E\left(\left[\max \left(v-p_{1 B}, 0-p_{1 A}\right)-\max \left(0, v-p_{2 B}\right)\right]^{+}\right)\right\}
\end{aligned}
$$

where $\rho$ is anticipated buying regret sensitivity coefficients with $\rho>0$, and $\sigma$ is anticipated waiting regret sensitivity coefficients with $\sigma>0$. We let $\gamma=\frac{\rho-\sigma}{1+\rho}$, and the larger $\gamma$ indicates the greater anticipated buying regret and the smaller anticipated waiting regret, and vice versa.

Based on the expected utility, consumers decide whether to purchase in advance and, if so, from which firm. If there is $E\left(u_{1 A}\right) \geq \max \left\{E\left(u_{1 B}\right), E\left(u_{W}\right)\right\}$, the consumer will purchase the retailer A's product in the first period, and if there is $E\left(u_{1 B}\right) \geq \max \left\{E\left(u_{1 A}\right), E\left(u_{W}\right)\right\}$, they will purchase the retailer B's product in the first period, and if there is $E\left(u_{W}\right)>\max \left\{E\left(u_{1 A}\right), E\left(u_{1 B}\right)\right\}$, they will wait to the second period in the first period. Because of all consumers predict their valuation and matching according to the same distributions, they are homogeneous in the first period which means that their choices are consistent.

Based on the utility function above, we first discuss the following four different competition case, and then find the competitive equilibrium: 
Case 1: $0 \leq p_{1 A} \leq v_{l}, \quad 0 \leq p_{1 B} \leq v_{l}$;

Case 2: $0 \leq p_{1 A}<v_{l}, \quad v_{l} \leq p_{1 B} \leq v_{h}$;

Case 3: $v_{l} \leq p_{1 A}<v_{h}, \quad 0 \leq p_{1 B}<v_{l}$;

Case 4: $v_{l} \leq p_{1 A}<v_{h}, \quad v_{l} \leq p_{1 B} \leq v_{h}$.

\subsection{The Profit of Firms}

The revenue function of retailer $i$ in the pre-sale period is:

$$
\pi_{1 i}= \begin{cases}0, & p_{1 i}>\bar{P}_{i j}^{k} \text { or } p_{1 i}>\bar{P}_{i W}^{k} ; \\ \frac{1}{2} p_{1 i}, & p_{1 A}=\bar{P}_{i j}^{k} \text { and } p_{1 i} \leq \bar{P}_{i W}^{k} ; \\ p_{1 i}, & p_{1 i}<\bar{P}_{i j}^{k} \text { and } p_{1 A} \leq \bar{P}_{i W}^{k},\end{cases}
$$

$k=\{1,2,3,4\}$ denotes the case $k$, and $\bar{P}_{i j}^{k}$ denotes that retailer is highest pre-sale price in case $k$ which satisfies $E\left(u_{1 i}\right) \geq E\left(u_{1 j}\right), \bar{P}_{i W}^{k}$ denotes that retailer $i$ s highest pre-sale price in case $k$ which satisfies $E\left(u_{1 i}\right) \geq E\left(u_{W}\right)$.

The revenue function in the spot period is:

$$
\pi_{2 A}=\left\{\begin{array}{l}
\alpha v_{l} N, \quad p_{2 A}=v_{l} \\
\frac{1}{2} \alpha v_{h} N, p_{2 A}=v_{h}
\end{array}, \quad \pi_{2 B}= \begin{cases}(1-\alpha) v_{l} N, & p_{2 B}=v_{l} \\
\frac{1}{2}(1-\alpha) v_{h} N, & p_{2 B}=v_{h}\end{cases}\right.
$$

$N$ represents the number of consumers waiting until the spot period.

The revenue function of whole selling period is:

$$
\pi_{i}=\pi_{1 i}+\pi_{2 i}
$$

\section{Analysis}

In this paper, we mainly focus on the sub-game perfect Nash equilibrium to solve the retailer's equilibrium prices and revenues.

In particular, since the retailers are symmetrical about $\alpha=\frac{1}{2}$, we only give the conclusion of Case 1 and Case 4 when consumers have the higher probability of matching for B's product, i.e. $0 \leq \alpha<\frac{1}{2}$. We can obtain the conclusion when $\frac{1}{2} \leq \alpha \leq 1$ by replacing A with B and replacing $\alpha$ with $1-\alpha$. Similarly, we only show the conclusion of Case 2, which are symmetrical to the conclusions of Case 3 about $\alpha=\frac{1}{2}$.

Therefore, the two retailers' pre-sale prices and revenues in three different cases are shown in the following Table 1 and Table 2.

Proposition 1: Comparing the revenues in three different cases,

1) when $0 \leq \alpha<\alpha^{1}$, then $\pi_{A}^{4} \geq \pi_{A}^{2} \geq \pi_{A}^{1}, \pi_{B}^{4} \geq \pi_{B}^{2} \geq \pi_{B}^{1}$;

2) when $\alpha^{1} \leq \alpha<\frac{1}{2}$, then $\pi_{B}^{4} \geq \pi_{B}^{1} \geq \pi_{B}^{2}$, and if

a) $\gamma \leq \gamma^{4}$, then $\pi_{A}^{2}>\pi_{A}^{4}=\pi_{A}^{1}$;

b) $\gamma^{4}<\gamma<\gamma^{1}$, then $\pi_{A}^{4}>\pi_{A}^{2}>\pi_{A}^{1}$; 
Table 1. The pre-sale prices in three different cases.

\begin{tabular}{|c|c|c|c|c|}
\hline Case $k$ & $\alpha$ & $\gamma$ & $p_{1 A}$ & $p_{1 B}$ \\
\hline \multirow{2}{*}{1} & $0<\alpha<\frac{1}{1}$ & $\gamma \leq \bar{\gamma}^{1}$ & 0 & $\min \left\{v_{l},(1-2 \alpha) E(v), \frac{(1-\alpha-\gamma) E(v)}{1-(1-\alpha) \gamma}\right\}$ \\
\hline & 2 & $\bar{\gamma}^{1}<\gamma<1$ & 0 & $\left(\max \left\{0, \frac{(1-\gamma-\alpha) E(v)}{1-\gamma+\alpha \gamma}\right\}, \min \left\{v_{l},(1-2 \alpha) E(v)\right\}\right)$ \\
\hline \multirow{3}{*}{2} & & $\gamma \leq \bar{\gamma}^{2}$ & 0 & $\min \left\{(1-2 \alpha) E(v), \frac{2(1-\alpha-\gamma \alpha) E(v)-(1-\alpha) \gamma v_{h}}{2-(1-\alpha) \gamma}\right\}$ \\
\hline & $0 \leq \alpha<\alpha^{1}$ & $\bar{\gamma}^{2}<\gamma<1$ & 0 & $\max \left\{v_{l}, \frac{2(1-\alpha-\gamma \alpha) E(v)-(1-\alpha) \gamma v_{h}}{2-(1-\alpha) \gamma}\right\},(1-2 \alpha) E(v)$ \\
\hline & $\alpha^{1} \leq \alpha<\frac{1}{2}$ & $\gamma<1$ & $v_{l}-(1-2 \alpha) E(v)$ & $v_{l}$ \\
\hline \multirow{2}{*}{4} & $0<0<1$ & $\gamma \leq \bar{\gamma}^{4}$ & $v_{l}$ & $\min \left\{v_{l}+(1-2 \alpha) E(v), \frac{2(1-\alpha) E(v)+\alpha \gamma v_{l}-\gamma v_{h}}{2-(1-\alpha) \gamma}\right\}$ \\
\hline & & $\bar{\gamma}^{4}<\gamma<1$ & $v_{l}$ & $\max \left\{v_{l}, \frac{2(1-\alpha) E(v)+\alpha \gamma v_{l}-\gamma v_{h}}{2-(1-\alpha) \gamma}\right\}, v_{l}+(1-2 \alpha) E(v)$ \\
\hline
\end{tabular}

Table 2. The revenues in three different cases.

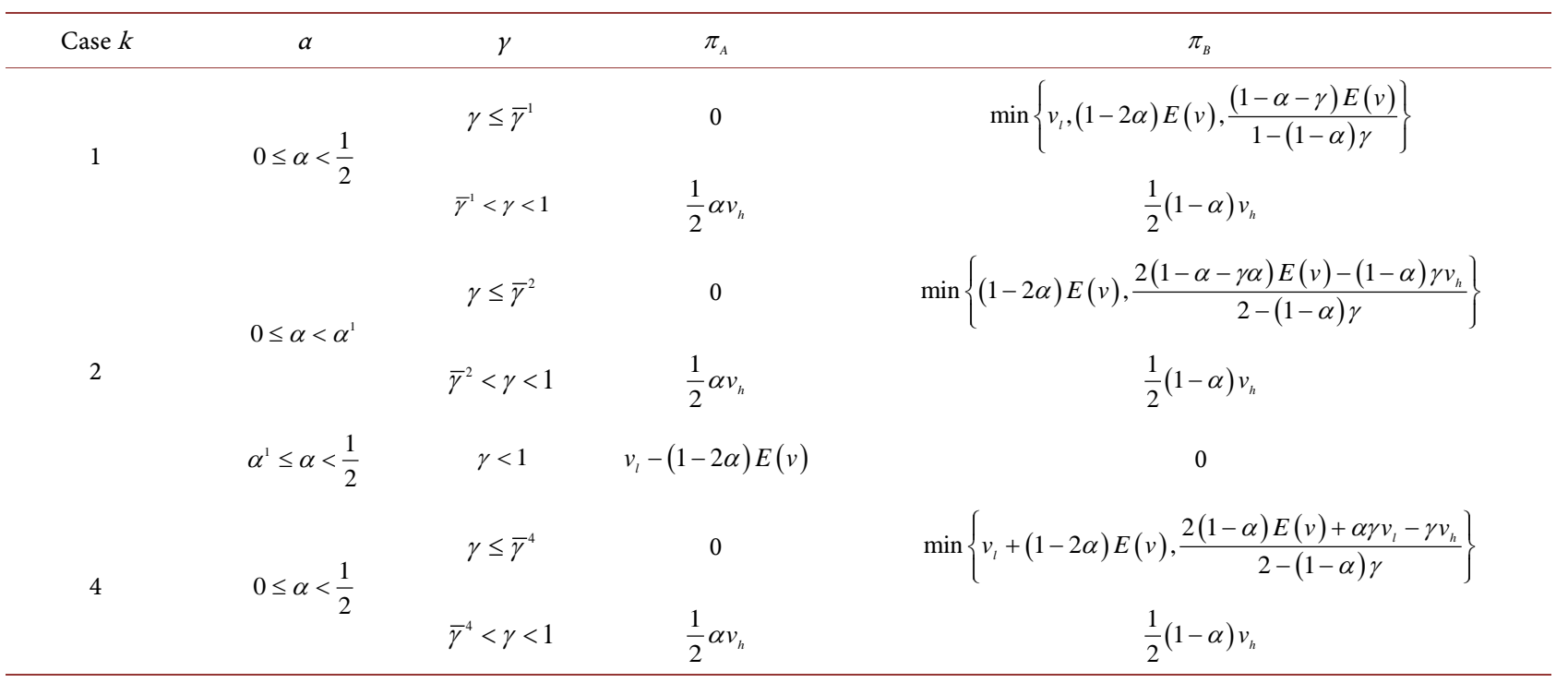

where $\alpha^{1}=\frac{E(v)-v_{l}}{2 E(v)}, \quad \bar{\gamma}^{1}=\max \left\{\frac{1}{3-2 \alpha}, \frac{(1-\alpha) E(v)-v_{l}}{E(v)-(1-\alpha) v_{l}}, \frac{(1-\alpha) v_{l}}{2 E(v)-(1-\alpha)^{2} v_{h}}\right\}$,

$\bar{\gamma}^{2}=\max \left\{\frac{2(1-\alpha)\left(2 E(v)-v_{h}\right)}{4 \alpha E(v)+2(1-\alpha) v_{h}-(1-\alpha)^{2} v_{h}}, \frac{2 \alpha E(v)}{\alpha v_{l}+v_{h}-(1-\alpha)(1-2 \alpha) E(v)}\right\}, \quad \bar{\gamma}^{4}=\max \left\{\frac{2\left(\alpha E(v)-v_{l}\right)}{v_{h}-v_{l}-(1-\alpha)(1-2 \alpha) E(v)}, \frac{2(1-\alpha)\left(2 E(v)-v_{h}\right)}{2 v_{h}-2 \alpha v_{l}-(1-\alpha)^{2} v_{h}}\right\}$

c) $\gamma^{1}<\gamma<1$, then $\pi_{A}^{4}=\pi_{A}^{1}>\pi_{A}^{2}$.

For retailer $i$ with higher probability of matching, the revenue obtained in the Case 4 is always the greatest, but for the retailer $j$ with lower probability of matching, only when the preference uncertainty is lower, or when the preference uncertainty is high and anticipated buying regret is high, the revenue in the Case 4 is the largest, otherwise, the revenue in the Case 2 is the largest. 
Proposition 2: with consumer anticipated regret, retailers' pre-sale competitive equilibrium is:

1) when $0 \leq \alpha<\alpha^{1}$, Case 4 is the unique Nash equilibrium;

2) when $\alpha^{1} \leq \alpha<\frac{1}{2}$, if

a) $\gamma<\gamma^{4}$, Case 1 is the unique Nash equilibrium

b) $\gamma>\gamma^{4}$, Case 4 is the unique Nash equilibrium;

Proposition 2 show that with the consumer anticipated regret, only the retailers simultaneously set high price or low price, can their competition achieve equilibrium. When the matching uncertainty is high and buying regret is low, the retailers will adopt low-price to compete, otherwise, they will adopt high-price to compete.

Proposition 3: 1) $\frac{\partial p_{1 B}^{1}}{\partial \gamma} \leq 0, \frac{\partial \pi_{B}^{1}}{\partial \gamma} \leq 0 ; \frac{\partial p_{1 A}^{1}}{\partial \gamma}=0, \frac{\partial \pi_{A}^{1}}{\partial \gamma} \geq 0 ;$

2) $\frac{\partial p_{1 B}^{4}}{\partial \gamma} \leq 0, \frac{\partial \pi_{B}^{4}}{\partial \gamma} \leq 0 ; \frac{\partial p_{1 A}^{4}}{\partial \gamma}=0, \frac{\partial \pi_{A}^{4}}{\partial \gamma} \geq 0$.

Sensitivity analysis of Nash equilibrium results found that the pre-sale price and revenue of retailer $i$ with higher probability of matching is non-increasing with anticipated buying regrets. The pre-sale price of retailer $j$ with lower probability of matching is not related to anticipated regret, yet the revenue presents a non-decreasing trend with anticipated buying regret. Anticipated waiting regret have the converse influence on pre-sale price and revenue.

\section{Conclusions}

We model the consumer utility function with anticipated regret in the pre-sale competition situation, and analyze the influence of anticipated regret on retailers' pre-sale prices and revenues. The results show that, for retailer with higher probability of matching, it is expected that anticipated buying regret is harm to improve revenue, while anticipated waiting regret is beneficial. Conversely, for the retailer with lower possibility of matching, anticipated buying regret is beneficial to improve revenue, while waiting regret is not. Thus, the retailer with higher probability of matching might benefit from inducing waiting regret like emphasizing a potentially forgone discount, and the retailer with lower possibility of matching might benefit from reducing waiting regret such as providing specific consumption time coupon or gifts to induce postponed purchase.

Considering the limitations of this paper, several related problems that need to be further explored. First, in this paper, we suppose the competitive retailers have no limitation on capacity, so future research might consider the retailer's capacity constraints into competition. Secondly, in our model, consumers' anticipated regret sensitivity coefficients are the same, it is more appreciate to investigate consumer regret heterogeneity in future research.

\section{References}

[1] Shugan, S.M. and Xie, J.H. (2000) Advance Pricing of Services and Other Implica- 
tions of Separating Purchase and Consumption. Journal of Service Research, 2, 227-239. https://doi.org/10.1177/109467050023001

[2] Shugan, S.M. and Xie, J.H. (2005) Advance-Selling as a Competitive Marketing Tool. International Journal of Research in Marketing, 22, 351-373. https://doi.org/10.1016/j.ijresmar.2004.11.004

[3] Xie, J.H. and Shugan, S.M. (2001) Electronic Tickets, Smart Cards, and Online Prepayments: When and How to Advance Sell. Marketing Science, 20, 219-243. https://doi.org/10.1287/mksc.20.3.219.9765

[4] Cooke, A.D.J., Meyvis, T. and Schwartz, A. (2008) Avoiding Future Regret in Purchase-Timing Decisions. Social Science Electronic Publishing, 27, 447-459.

[5] Simonson, I. (1992) The Influence of Anticipating Regret and Responsibility on Purchase Decisions. Journal of Consumer Research, 19, 105-18. https://doi.org/10.1086/209290

[6] Diecidue, E., Rudi, N. and Tang, W. (2011) Dynamic Purchase Decisions under Regret: Price and Availability. Decision Analysis, 9, 22-30. https://doi.org/10.1287/deca.1110.0227

[7] Nasiry, J. and Popescu, I. (2012) Pre-sale When Consumers Regret. Management Science, 58, 1160-1177. https://doi.org/10.1287/mnsc.1110.1473

[8] Chao, Y. and Liu, L. and Zhan, D.Y. Vertical Probabilistic Selling under Competition: The Role of Consumer Anticipated Regret. NET Institute Working Paper No. 16-14.

[9] Jiang, B.J., Narasimhan, C. and Turut, Ö. (2017) Anticipated Regret and Product Innovation. Management Science, 63, 4308-4323.

https://doi.org/10.1287/mnsc.2016.2555 


\section{Appendix. Proofs}

Proof of Proposition 1: 1) when $0 \leq \alpha<\alpha^{1}$, as for $\pi_{A}$, if $\gamma \leq \gamma^{4}$, $\pi_{A}^{4}=\pi_{A}^{2}=\pi_{A}^{1}=0$, if $\gamma^{4}<\gamma \leq \gamma^{2}, \pi_{A}^{4}=\frac{1}{2} \alpha v_{h}, \pi_{A}^{2}=\pi_{A}^{1}=0$, if $\gamma^{2}<\gamma \leq \gamma^{1}$, $\pi_{A}^{4}=\pi_{A}^{2}=\frac{1}{2} \alpha v_{h}, \pi_{A}^{1}=0$, otherwise, $\pi_{A}^{4}=\pi_{A}^{2}=\pi_{A}^{1}=\frac{1}{2} \alpha v_{h}$.

As for $\pi_{B}$, if $\gamma \leq \gamma^{4}$,

$\pi_{B}^{4}=\min \left\{v_{l}+(1-2 \alpha) E(v), \frac{2(1-\alpha) E(v)+\alpha \gamma v_{l}-\gamma v_{h}}{2-(1-\alpha) \gamma}\right\} \in\left(v_{l}, v_{h}\right)$,

$\pi_{B}^{2}=\min \left\{(1-2 \alpha) E(v), \frac{2(1-\alpha-\gamma \alpha) E(v)-(1-\alpha) \gamma v_{h}}{2-(1-\alpha) \gamma}\right\}>v_{l}$,

$v_{l}+(1-2 \alpha) E(v)>(1-2 \alpha) E(v)$,

$\frac{2(1-\alpha) E(v)+\alpha \gamma v_{l}-\gamma v_{h}}{2-(1-\alpha) \gamma}>\frac{2(1-\alpha-\gamma \alpha) E(v)-(1-\alpha) \gamma v_{h}}{2-(1-\alpha) \gamma}$, so, $\pi_{B}^{4}>\pi_{B}^{2}$,

$\pi_{B}^{1}=\min \left\{v_{l},(1-2 \alpha) E(v), \frac{(1-\alpha-\gamma) E(v)}{1-(1-\alpha) \gamma}\right\} \in\left(0, v_{l}\right)$, therefore, $\pi_{B}^{4}>\pi_{B}^{2}>\pi_{B}^{1} ;$

if $\gamma^{4}<\gamma \leq \gamma^{2}$,

$\pi_{B}^{4}=\frac{1}{2}(1-\alpha) v_{h}>\min \left\{v_{l}+(1-2 \alpha) E(v), \frac{2(1-\alpha) E(v)+\alpha \gamma v_{l}-\gamma v_{h}}{2-(1-\alpha) \gamma}\right\} \quad, \quad \pi_{B}^{2}$

and $\pi_{B}^{1}$ are same to the case when $\gamma \leq \gamma^{4}$, so $\pi_{B}^{4}>\pi_{B}^{2}>\pi_{B}^{1}$; if $\gamma^{2}<\gamma \leq \gamma^{1}$,

$\pi_{B}^{4}=\pi_{B}^{2}=\frac{1}{2}(1-\alpha) v_{h}>\min \left\{(1-2 \alpha) E(v), \frac{2(1-\alpha-\gamma \alpha) E(v)-(1-\alpha) \gamma v_{h}}{(2-(1-\alpha) \gamma)}\right\}$, so

$>v_{l}>\pi_{B}^{1}=\min \left\{v_{l},(1-2 \alpha) E(v), \frac{(1-\alpha-\gamma) E(v)}{1-(1-\alpha) \gamma}\right\} \in\left(0, v_{l}\right)$

$\pi_{B}^{4}=\pi_{B}^{2}>\pi_{B}^{1}$; otherwise, $\pi_{B}^{4}=\pi_{B}^{2}=\pi_{B}^{1}=\frac{1}{2}(1-\alpha) v_{h}$.

2) when $0 \leq \alpha<\alpha^{1}, \pi_{B}^{2}=0, \pi_{B}^{4}$ and $\pi_{B}^{1}$ are same to the case when $0 \leq \alpha<\alpha^{1}$, so $\pi_{B}^{4} \geq \pi_{B}^{1}>\pi_{B}^{2}$. As for $\pi_{A}$, if $\gamma \leq \gamma^{4}$, $\pi_{A}^{2}=v_{l}-(1-2 \alpha) E(v)>\pi_{A}^{4}=\pi_{A}^{1}=0$, if $\gamma^{4}<\gamma \leq \gamma^{1}$, $\pi_{A}^{4}=\frac{1}{2} \alpha v_{h}>\pi_{A}^{2}=v_{l}-(1-2 \alpha) E(v)>\pi_{A}^{1}=0 ;$ if $\gamma^{1}<\gamma<1$, $\pi_{A}^{4}=\pi_{A}^{1}=\frac{1}{2} \alpha v_{h}>\pi_{A}^{2}=v_{l}-(1-2 \alpha) E(v)$.

Proof of Proposition 2. According to proposition 1, and using game methods, when $0 \leq \alpha<\alpha^{1}$, or $\alpha^{1} \leq \alpha<\frac{1}{2}$ and $\gamma>\gamma^{4}$, it is obvious the retailers' revenues are the most in Case 4, Case 4 is their respective optimal strategy with given another's strategy, so, Case 4 is the Nash equilibrium. When $\alpha^{1} \leq \alpha<\frac{1}{2}$ and $\gamma<\gamma^{4}$, only Case 1 is fit for the equilibrium condition, while another retailer might reduce price to obtain more revenue given one retailer's strategy in the 
other cases.

Proof of Proposition 3. 1) when $\gamma \leq \bar{\gamma}^{1}$, if

$\min \left\{v_{l},(1-2 \alpha) E(v), \frac{(1-\alpha-\gamma) E(v)}{1-(1-\alpha) \gamma}\right\}=\min \left\{v_{l},(1-2 \alpha) E(v)\right\}$, then $\frac{\partial p_{1 B}^{1}}{\partial \gamma}=0$, $\frac{\partial \pi_{B}^{1}}{\partial \gamma}=0$, otherwise, $\frac{\partial p_{1 B}^{1}}{\partial \gamma}=\frac{(-2+\alpha) \alpha \mu}{(1+(-1+\alpha) \gamma)^{2}}<0, \frac{\partial \pi_{B}^{1}}{\partial \gamma}=\frac{(-2+\alpha) \alpha \mu}{(1+(-1+\alpha) \gamma)^{2}}<0$, when $\gamma>\bar{\gamma}^{1}, \frac{\partial p_{1 B}^{1}}{\partial \gamma}=0, \frac{\partial \pi_{B}^{1}}{\partial \gamma}=0 ; \frac{\partial p_{1 A}^{1}}{\partial \gamma}=0$, when $\gamma \neq \bar{\gamma}^{1}, \frac{\partial \pi_{A}^{1}}{\partial \gamma}=0$, otherwise, $\frac{\partial \pi_{A}^{1}}{\partial \gamma}>0$.

2) when $\gamma \leq \bar{\gamma}^{4}$, if

$\min \left\{v_{l},(1-2 \alpha) E(v), \frac{2(1-\alpha) E(v)+\alpha \gamma v_{l}-\gamma v_{h}}{2-(1-\alpha) \gamma}\right\}=\min \left\{v_{l},(1-2 \alpha) E(v)\right\}$, then $\frac{\partial p_{1 B}^{4}}{\partial \gamma}=0, \frac{\partial \pi_{B}^{4}}{\partial \gamma}=0$, otherwise, $\frac{\partial p_{1 B}^{4}}{\partial \gamma}=\frac{\partial \pi_{B}^{4}}{\partial \gamma}=\frac{2\left((-1+\alpha)^{2} \mu-v_{h}+\alpha v_{l}\right)}{(2+(-1+\alpha) \gamma)^{2}}<0$, when $\gamma>\bar{\gamma}^{4}, \frac{\partial p_{1 B}^{4}}{\partial \gamma}=0, \frac{\partial \pi_{B}^{4}}{\partial \gamma}=0 ; \frac{\partial p_{1 A}^{4}}{\partial \gamma}=0$, when $\gamma \neq \bar{\gamma}^{4}, \frac{\partial \pi_{A}^{4}}{\partial \gamma}=0$, otherwise, $\frac{\partial \pi_{A}^{4}}{\partial \gamma}>0$. 